\title{
Toints
} $\sqrt{10}$

\section{Correlation between body mass index and quadrupled hamstring tendon autograft size in ACL reconstruction}

\author{
ZAFER ATBAŞI ${ }^{1}$, ERSIN ERÇIN ${ }^{2}$, YUSUF ERDEM ${ }^{3}$, TULUHAN YUNUS EMRE ${ }^{4}$, \\ HALIS ATIL ATILLA ${ }^{1}$, ADEM PARLAK ${ }^{5}$ \\ 1 Department of Orthopaedic Surgery, Ankara Mevki Military Hospital, Ankara, Turkey \\ 2 Department of Orthopaedics and Traumatology, Bakirkoy Dr. Sadi Konuk Training and Research Hospital, Istanbul, Turkey \\ ${ }^{3}$ Department of Orthopaedics and Traumatology, Gulhane Military Academy, Ankara, Turkey \\ ${ }^{4}$ Department of Orthopaedics and Traumatology, Acibadem Hospital, Istanbul, Turkey \\ ${ }^{5}$ President Guard Regimen, Ankara, Turkey
}

\begin{abstract}
Purpose: the aim of this study was to assess the relationship of patient weight, height and body mass index (BMI) with the size of the quadrupled hamstring tendon used in anterior cruciate ligament (ACL) reconstruction.

Methods: from patient records, we retrospectively assessed the weight, height, BMI and graft sizes of 126 patients who underwent ACL reconstruction using a quadrupled hamstring tendon autograft between January 2010 and January 2013 at our institution. The data obtained from perioperative measurements were studied using correlation analysis.

Results: statistically significant relationships were found between patient height and graft diameter $(\phi=$ $0.033, r=0.19)$ and between patient weight and graft diameter $(p<0.0001, r=0.33)$. No statistically significant relationships were found between graft diameter and BMI or between graft length and patient height, weight and BMI $(p>0.05)$.

Conclusions: patient height and weight were found to be related to quadrupled hamstring graft diameter in our patient population. BMI was not related to the quadrupled hamstring graft size. The exact size of the
\end{abstract}

\footnotetext{
Corresponding Author:

Ersin Erçin, MD

Department of Orthopaedics and Traumatology, Bakirkoy

Dr. Sadi Konuk Training and Research Hospital

Tevfik Saglam cad. no: 11, 34147, Zuhuratbaba,

Istanbul, Turkey

E-mail: ersine@hotmail.com
}

graft cannot be predicted preoperatively on the basis of these variables.

Level of evidence: Level IV, retrospective case series.

Keywords: body mass index, hamstring, tendon size, anterior cruciate ligament, reconstruction.

\section{Introduction}

The anterior cruciate ligament (ACL) is an important structural component for knee stabilization. Although it is quite strong, it is vulnerable to several injury mechanisms, in particular, high-energy tibial torsion during contact sports $(1,2)$. The increased practice of sporting activities has led to an increase in the prevalence of ACL injuries over the past 25 years, and continual advances in surgical techniques have rendered arthroscopic ACL reconstruction a common orthopedic procedure (3).

Although patellar tendon autografts are used in this procedure with great success, hamstring autografts have recently become more popular; they are preferred to bone-patella-tendon autografts because of the lower risk of donor site morbidity. In addition, it is widely accepted that four-strand autografts are the strongest option in ACL reconstruction (4). However, the variation in graft size from patient to patient is recognized as one of the most critical aspects of this procedure, and preoperative prediction of the graft size is important in the preparation of the surgery (5).

Therefore, many attempts have been made to develop a method for hamstring size prediction using several cor- 
relations. Our hypothesis is that hamstring graft size differs with differences in patient height, weight and body mass index (BMI). In the present study, we aimed to assess the relationship between these anthropometric parameters and hamstring autograft size.

\section{Methods}

After obtaining permission from our institution's investigational review board, patients submitted to ACL reconstruction using a quadrupled (four-strand) hamstring tendon autograft between January 2010 and January 2013 were included in this study. All the included patients were male military personnel at the time of the operation. Patients who had previously undergone knee surgery or had experienced fractures around the knee were excluded. The weight, height and BMI of each patient had been recorded preoperatively.

The same graft harvesting procedure was followed in all the patients. The knees were covered and positioned in $90^{\circ}$ flexion. After palpating and marking the anatomical landmarks over the knee, a 5-cm oblique anterior skin incision was performed $1 \mathrm{~cm}$ medial and inferior to the tibial tuberosity over the pes anserinus insertion site. After reaching the pes anserinus, the sartorial fascia was incised parallel to the skin incision. First the gracilis and then the semitendinosus tendons were removed from their insertion sites by sharp dissection. Both ends of the tendon grafts were stitched using the Krackow technique with nonabsorbable no. 2 sutures. An open tendon stripper was used to split the proximal musculotendinous junction. Absorbable no. 3.0 sutures were used to keep the quadrupled shape stable. Quadrupled graft lengths were measured using a ruler without applying any tension (Fig. 1). The graft diameter, defined as the smallest cylindrical dimension that could pass through the femoral tunnel, was determined using sizing cylinders with increments of $1 \mathrm{~mm}$ (Fig. 2). In each patient, the transtibial technique was used, and a minimum of $25 \mathrm{~mm}$ of the quadrupled graft was placed within the femoral tunnel.

The data obtained from the perioperative measurements were analyzed using SPSS version 16 . The height, weight and BMI of the patients were entered into the SPSS program to look for correlations between the clinical data and the intraoperatively measured graft length and diameter. Pearson's correlation coefficients were calculated to evaluate any possible associations between anthropometric characteristics and intraoperatively measured hamstring graft lengths and diameters. A $p$ value of 0.05 was considered statistically significant.

\section{Results}

In total, 126 male patients with a mean age of $24.2 \pm$ 4.65 years who underwent ACL reconstruction surgery were included in this study. The operation was performed on 56 left knees and 70 right knees. The patients had a mean height of $176.28 \pm 5.40 \mathrm{~cm}$ and a mean weight of $77.91 \pm 8.13 \mathrm{~kg}$, while their mean BMI was $25.07 \pm 2.28 \mathrm{~kg} / \mathrm{m}^{2}$. The mean graft diameter was $8.14 \pm 0.61$ and the mean graft length was $13.25 \pm 1.18$.

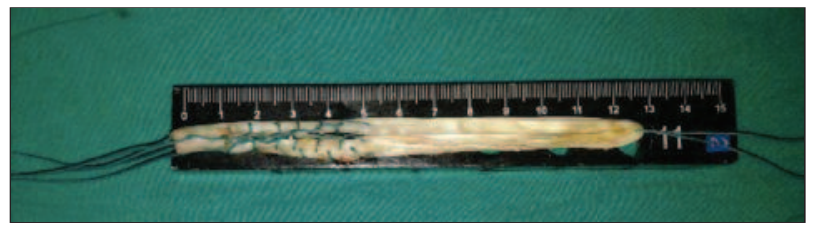

Figure 1. Graft length measurement with ruler. Intraoperative photograph of quadrupled hamstring tendons (gracilis and semitendinosus tendons).

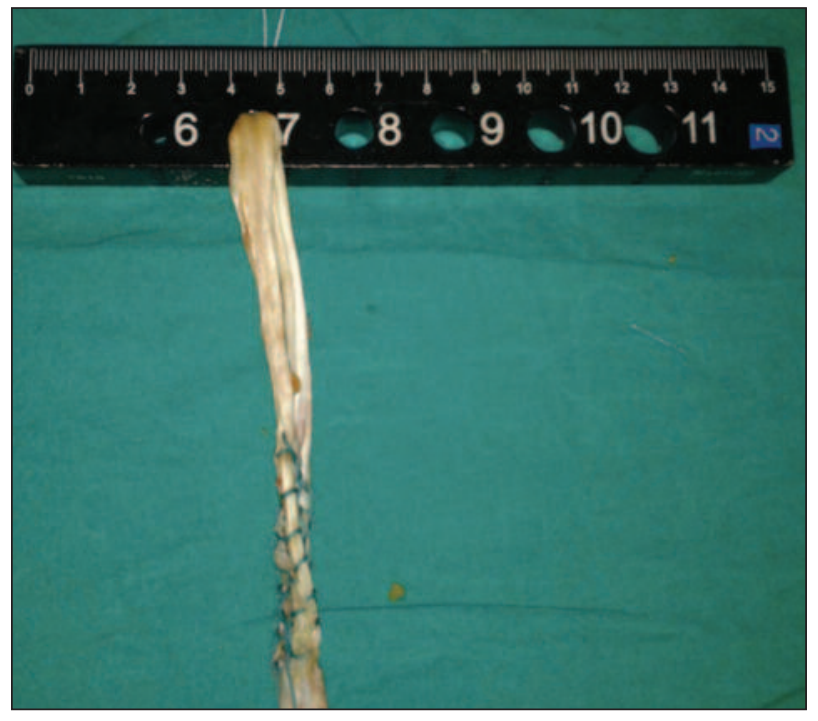

Figure 2. Graft diameter measurement. Intraoperative photograph of quadrupled hamstring tendons (gracilis and semitendinosus tendons). 
A statistically significant relationship was found between patient height and graft diameter $(p=0.033, r$ $=0.19)$. Similarly, a statistically significant relationship was found between patient weight and graft diameter $(p<0.0001, r=0.33)$ (Fig. 3). There was no statistically significant relationship between BMI and graft diameter $(p>0.05)$, or between any of the anthropometric parameters (height, weight and BMI) and graft length $(p>0.005)$.

\section{Discussion}

ACL reconstruction with quadrupled hamstring tendons has increased in popularity among orthopedic surgery procedures (6). Biomechanical studies have shown that quadrupled hamstring tendon strength ranges from $4108 \mathrm{~N}$ to $4213 \mathrm{~N}$ (7). These values are twice as high as those of native ACL; thus, these grafts are more than adequate. Graft diameter and length are important for proper graft placement and durability. The diameter of the hamstring graft varies from patient to patient, and it is difficult to gauge or predict its size preoperatively (5).

Several studies have tried to show a relationship between anthropometric measurements and graft size. Thomas et al. (8) measured these parameters in 121 patients and reported no statistically significant corre- lation between BMI and tendon size. Tuman et al. (9) reported that hamstring graft diameter is correlated with patient height, weight, age and sex. They also stated that height in both genders could be used to predict the graft diameter; moreover, neither BMI nor graft length was found to be correlated with graft length. Since our patient group consisted only of young males, we did not investigate correlations with age and sex; indeed, these two parameters were our fixed variables.

Like the above-mentioned studies, we did not find any correlation between BMI and graft size (diameter and length). By contrast, Papastergiou et al. (2), evaluating 61 patients who underwent ACL reconstruction, found a moderate correlation between semitendinosus and gracilis graft diameter and patient height and weight. They also reported a correlation with BMI.

Park et al. (10), in a study of 296 patients, found the strongest correlation between graft diameter and patient height, while Ma et al. (11), in an extensive study of 536 male patients, found patient height and age to be correlated with the tendon diameter and emphasized that these variables could be used preoperatively to predict the tendon length.

Graft diameter is known to differ between the genders: males tend to have larger grafts than females. In addition, graft diameter tends to increase with height in males (12). Çeliktaş et al. (13) studied an all-male
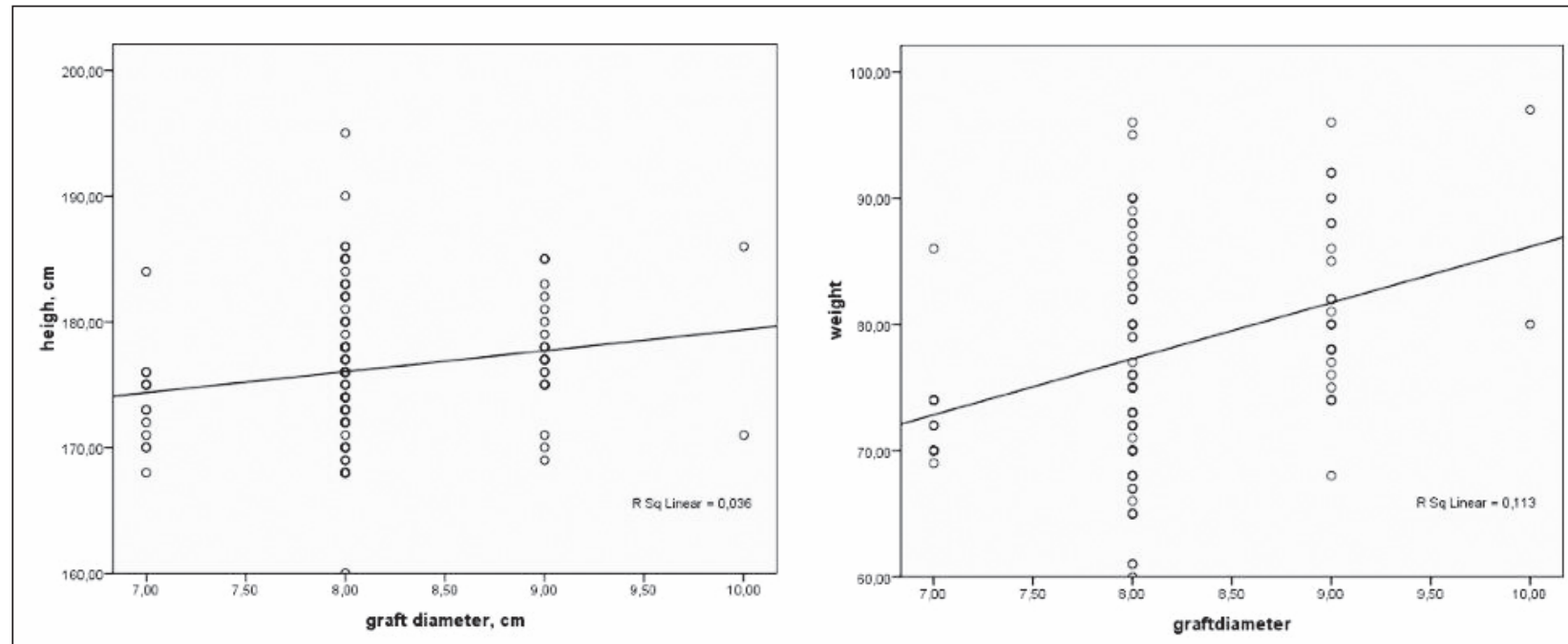

Figure 3. Statistical graphics of patient height-graft diameter and patient weight-graft diameter. The formula of height and graft diameter $y=13,33$. $X+66,66$. The formula of weight and graft diameter $y=13,33$. $X-33,33$. 
group and found results similar to ours. The finding of a relatively higher risk of small and thin graft size in females has been shown to be associated with poorer outcomes (14). While we could not make comparisons with females, within our population of healthy male military personnel, all the grafts were adequate for use in ACL reconstruction. In some studies, patient height has been found to correlate with graft length; however, the relationship between patient height and graft size remains controversial $(2,8,10)$. In our study, we did not measure the grafts separately. We measured the quadrupled graft of the semitendinosus and gracilis together and did not find any significant relationships between graft length and any other variable. Measuring the grafts together is one of the weak points of our study because it meant that we could not obtain separate data for the tendons.

Some studies advocate the use of radiological measurements preoperatively, and cross-sectional areas of the hamstring tendons in MRI studies have shown significant correlations with intraoperative graft measurements (15). Three-dimensional computed tomography (16) and ultrasonography (17) have also been used to predict graft size. However, these radiological assessments are weakened by lack of standardization of measurement, intra-observer and inter-observer variability, and the need for a trained radiologist.

In this study, we tried to assess relations between quadrupled hamstring graft size and anthropometric measurements, and only patient height and weight were found to show a relationship with graft diameter. Graft length did not seem to correlate with patient height, weight or BMI. Moreover, BMI was not found to be related to graft length or diameter and therefore should not be used to determine graft size. Although many studies have found that patient weight and height are correlated with graft diameter, no single anthropometric measurement has been found that is adequate for predicting graft size preoperatively. In future, more studies combining clinical and radiological data could be helpful to predict graft size more precisely.

In conclusion, patient height and weight were found to be correlated to the quadruple hamstring graft diameter in our patient population. Body mass index is not an adequate predictor of quadrupled hamstring graft size.

The exact size of the graft cannot be predicted preoperatively on the basis of these variables.

\section{References}

1. Lephart SM, Kocher MS, Harner CD, et al. Quadriceps strength and functional capacity after anterior cruciate ligament reconstruction. Am J Sports Med. 1993;21:738-743.

2. Papastergiou SG, Konstantinidis GA, Natsis K, et al. Adequacy of semitendinosus tendon alone for anterior cruciate ligament reconstruction graft and prediction of hamstring graft size by evaluating simple anthropometric parameters. Anat Res Int. 2012;2012:424158.

3. Loo WL, Liu BYE, Lee YHD, et al. Can we predict ACL hamstring graft sizes in the Asian male? A clinical relationship study of anthropometric features and 4-strand hamstring graft sizes. Malays Orthop J. 2010;4:9-12.

4. Pinczewski LA, Lyman J, Salmon LJ, et al. A 10-year comparison of anterior cruciate ligament reconstructions with hamstring tendon and patellar tendon autograft: a controlled, prospective trial. Am J Sports Med. 2007;35:564-574.

5. Ma CB, Keifa E, Dunn W, et al. Can pre-operative measures predict quadruple hamstring graft diameter? Knee. 2010; $17: 81-83$.

6. Schwartzberg R, Burkhart B, Lariviere C. Prediction of hamstring tendon autograft diameter and length for anterior cruciate ligament reconstruction. Am J Orthop (Belle Mead NJ). 2008;37:157-159.

7. Butler RJ, Dai B, Garrett WE, et al. Changes in landing mechanics in patients following anterior cruciate ligament reconstruction when wearing an extension constraint knee brace. Sports Health. 2014;6:203-209.

8. Thomas S, Bhattacharya R, Saltikov JB, et al. Influence of anthropometric features on graft diameter in ACL reconstruction. Arch Orthop Trauma Surg. 2013;133:215-218.

9. Tuman JM, Diduch DR, Rubino LJ, et al. Predictors for hamstring graft diameter in anterior cruciate ligament reconstruction. Am J Sports Med. 2007;35:1945-1949.

10. Park SY, Oh H, Park S, et al. Factors predicting hamstring tendon autograft diameters and resulting failure rates after anterior cruciate ligament reconstruction. Knee Surg Sports Traumatol Arthrosc. 2013;21:1111-1118.

11. Ma CB, Keifa E, Dunn W, et al. Can pre-operative measures predict quadruple hamstring graft diameter? Knee. 2010; 17:81-83.

12. Hill PF, Russell VJ, Salmon LJ, et al. The influence of supplementary tibial fixation on laxity measurements after anterior cruciate ligament reconstruction with hamstring tendons in female patients. Am J Sports Med. 2005;33:94-101.

13. Celiktaş M, Gölpinar A, Köse Ö, et al. Prediction of the quadruple hamstring autograft thickness in ACL reconstruction using anthropometric measures. Acta Orthop Traumatol Turc. 2013;47:14-18.

14. Chandrashekar N, Slauterbeck J, Hashemi J. Sex-based differences in the anthropometric characteristics of the anterior cruciate ligament and its relation to intercondylar notch geometry: a cadaveric study. Am J Sports Med. 2005;33:14921498.

15. Beyzadeoglu T, Akgun U, Tasdelen N, et al. Prediction of semitendinosus and gracilis autograft sizes for ACL reconstruction. Knee Surg Sports Traumatol Arthrosc. 2012; 20: 1293-1297.

16. Yasumoto M, Deie M, Sunagawa T, et al. Predictive value of preoperative 3-dimensional computer tomography measurement of semitendinosus tendon harvested for anterior cruciate ligament reconstruction. Arthroscopy. 2006;22:259-264.

17. Erquicia JI, Gelber PE, Doreste JL, et al. How to improve the prediction of quadrupled semitendinosus and gracilis autograft sizes with magnetic resonance imaging and ultrasonography. Am J Sports Med. 2013;41:1857-1863. 